
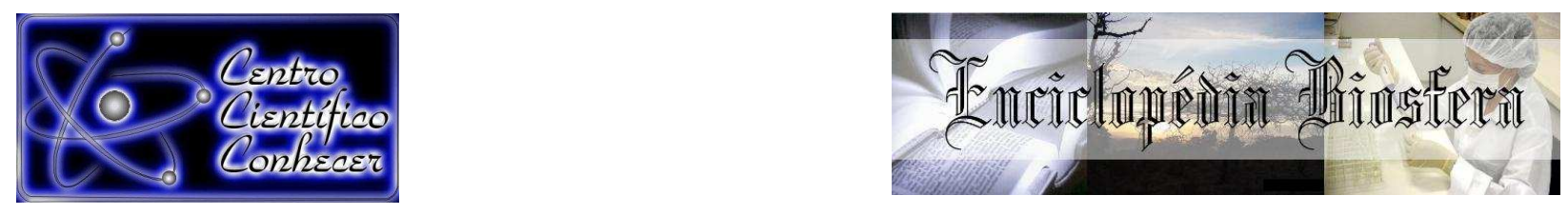

\title{
OCORRÊNCIA DE ENTEROPARASITOSES EM CRIANCAS ATENDIDAS PELO PROGRAMA DE ERRADICAÇÃO DO TRABALHO INFANTIL (PETI) NOS DISTRITOS DE INTERLÂNDIA E SOUSÂNIA NA CIDADE DE ANAPOLIS-GO NO ANO DE 2013
}

\author{
Rafael Sousa Silva Crozara ${ }^{1}$, Jaqueline Rodrigues da Silva ${ }^{1}$, Weslei Antonio \\ Pereira $^{1}$, Renan de Souza Soares ${ }^{2}$, Ariana Alves Rodrigues ${ }^{3}$. \\ 1 Bacharel em Biomedicina. Anhanguera Educacional. \\ 2 Mestrando em Ciências Ambientais, Universidade Federal de Goiás. \\ 3 Doutoranda em Ciências Ambientais, Universidade Federal de Goiás \\ (ariana.biomed@gmail.com)
}

Recebido em: 08/04/2016 - Aprovado em: 30/05/2016 - Publicado em: 20/06/2016 DOI: 10.18677/Enciclopedia_Biosfera_2016_076

\begin{abstract}
RESUMO
As enteroparasitoses representam um grave problema de saúde pública, sendo as crianças em idade escolar as mais acometidas. O estudo teve como objetivo estabelecer a ocorrência de parasitoses intestinais em crianças atendidas pelo PETI (Programa de erradicação do trabalho infantil) nos distritos de Interlândia e Sousânia no ano de 2013. Foram avaliadas 48 crianças de 05 a 14 anos de idade atendidas pelo PETI. De cada criança foram coletadas três amostras de fezes em dias alternados e a análise parasitológica foi feita pelo método de Hoffman. Da população estudada $37,5 \%$ apresentava algum tipo de parasitose. Das amostras analisadas foram encontradas somente protozoários, sendo identificados Entamoeba coli 44\%, Endolimax nana 22,5\%, Giardia lamblia 22,5\%, Entamoeba histolytica 11\%. Sugerese que os resultados obtidos sejam resposta da fragilidade das circunstâncias de saneamento básico, e situações de moradia e costumes de asseio das crianças estudadas nos distritos de Intêrlandia e Sousânia.
\end{abstract}

PALAVRAS-CHAVE: crianças, enteroparasitoses, protozoários.

\section{OCCURRENCE OF ENTEROPARASITOSIS IN CHILDREN ATTENDEDING CHILD LABOR ERRADICACION PROGRAM IN DISTRICTS OF INTERLÂNDIA AND SOUSÂNIA IN ANÁPOLIS-GO IN THE YEAR OF 2013}

\begin{abstract}
The enteroparasitosis represent a serious public health issue, and the school age children are the most affected. The study aimed to establish the occurrence of intestinal parasites in children assisted by PETI (child labor eradication program) in the districts of Interlândia and Sousânia in 2013. Forty eight children of 05 to 14 years old assisted by PETI were evalueted. From each child was collected three stool samples on alternate days and parasitological analysis was done by Hoffman method. Of the population analyzed, $37.5 \%$ had some type of parasitosis . In the samples were found only protozoan,such as Entamoeba coli 44\%, Endolimax nana
\end{abstract}


$22.5 \%$, Giardia lamblia $22.5 \%$, Entamoeba histolytica $11 \%$. It is suggested that the results obtained are a response to the fragility of basic sanitation conditions, housing situations and neatness customs of children from the districts of Intêrlandia and Sousânia.

KEYWORDS: Enteroparasitosis, kids, protozoan.

\section{INTRODUÇÃO}

As enteroparasitoses constituem um grave problema de saúde pública de cunho mundial. Segundo dados da Organização Mundial da Saúde (OMS), as doenças infecciosas e parasitárias persistem a destacar-se entre as principais circunstâncias de óbito, tornando-se responsáveis por dois a três milhões de mortes por ano. No Brasil, essas patologias ocorrem nas variadas regiões do país, seja em zona rural ou urbana e em diversas faixas etárias (SILVA et al., 2011a; VASCONCELOS et al., 2011).

A prevalência de enteroparasitoses é um dos mais adequados indicadores das condições socioeconômicas e podem estar vinculadas a vários fatores, como: instalações sanitárias inapropriadas, contaminação fecal da água e de alimentos ingeridos, elementos socioculturais, convívio com animais, saneamento básico, ciclos biológicos e do tipo de parasito contaminante (BELO et al., 2012).

As más condições de saneamento implicam diretamente no aparecimento de doenças parasitárias, especialmente as enteroparasitoses. $O$ termo enteroparasitose refere-se a parasitas intestinais cujos agentes etiológicos são helmintos ou protozoários, e que em pelo menos uma das etapas do ciclo evolutivo, encontram-se no sistema digestório do homem, acarretando diversas alterações patológicas (GOMES et al., 2011).

No Brasil, $36,1 \%$ das residências não são abastecidas de água por rede geral, $7,2 \%$ do volume de água fornecida não recebe tratamento e $47,8 \%$ dos municípios não contam com esgotamento sanitário, constituindo o serviço de menor abrangência nos municípios (SILVA et al., 2011b). No Norte Goiano há insuficiência de dados a respeito da prevalência de enteroparasitoses nos habitantes, especialmente em crianças. É relevante destacar que pesquisas de prevalência de parasitos intestinais são escassas no estado de Goiás (GELATTI et al., 2013).

Existem poucos dados autênticos a respeito da prevalência dessas parasitoses, que em geral se fundamentam em estimativas e na maior parte dos casos é subnotificado por parte dos atendimentos de atenção básica a saúde, levando a índices duvidosos (MORAIS NETO et al., 2009). Entretanto, nos últimos 30 anos, observou-se uma diminuição da prevalência de enteroparasitoses. Em algumas regiões em desenvolvimento são encontradas taxas de infecção próxima de $30 \%$, principalmente em se tratando da ocorrência de pelo menos uma monoparasitose (ANTUNES et al., 2011).

Três elementos são fundamentais para que aconteça a transmissão das enteroparasitoses: o estado do hospedeiro, o parasito e o meio ambiente. Referente ao hospedeiro destacam-se: a idade, estado nutricional, fatores genéticos, culturais, habituais e profissionais. Cabe ao parasito escapar das alterações bioquímicas e imunológicas desencadeadas pelo organismo em resposta ao parasito. Por fim, as circunstâncias ambientais referem-se aos fatores anteriores a infecção, tais como boas condições sanitárias, bons hábitos de higiene, aspectos culturais e sociais (FREl et al., 2008). 
As enteroparasitoses são provocadas, em geral, por protozoários como Giardia lamblia e Entamoeba histolytica, platelmintos como Taenia solium, Taenia saginata e Hymenolepis nana e nematódeos como Trichuris trichiura, Strongyloides stercoralis, Enterobius vermiculares, Ascaris lumbricoides, Ancylostoma duodenale e Necator americanus. Essas infecções provocam um amplo espectro de sintomas com características comuns (BARRETO et al., 2012).

As manifestações clínicas mais frequentes causadas no hospedeiro são diarreia, sangramento gastrointestinal, anemia, perda de peso, dores abdominais, ansiedade, inquietação e, em situações críticas a morte. Esses sintomas variam de acordo com a carga parasitária, defesas do hospedeiro e a fase do ciclo biológico. A maioria das enteroparasitoses é transmitida por via alimentar (BELLIN \& GRAZZIOTIN, 2011).

As crianças em idade escolar são as mais acometidas e mais prejudicadas pelas enteroparasitoses, uma vez que suas práticas de higiene são, na maioria das vezes, inapropriadas e a imunidade ainda não está completamente eficaz para a extinção dos parasitas (MELO et al., 2010). Os enteroparasitas são principalmente veiculados por meio de alimentos e água contaminada. As mãos das pessoas podem conduzir as formas infectantes, sejam de cistos de protozoários ou ovos de helmintos. A contaminação humana é mais comum em crianças, por meio da via oral-fecal, sendo águas e alimentos contaminados os principais veículos de transmissão (MONROE et al., 2013).

Para o controle das parasitoses intestinais são indispensáveis investimentos em saneamento básico e educação. Contudo, ações mais efetivas para melhoria da qualidade de vida sejam implementadas, considera-se importante à execução de exames parasitológicos para o diagnóstico correto e terapia adequada destas doenças (CANTUÁRIA et al., 2011). Em consequência dos efeitos prejudiciais à saúde das pessoas e, especialmente, dos impactos financeiros, vários projetos têm sido conduzidos para o monitoramento das parasitoses intestinais em distintos países, mas, verifica-se divergência entre o resultado alcançado nos países mais desenvolvidos e aquele constatado nas economias em desenvolvimento. Além do aspecto econômico das medidas técnicas, a falta de programas educativos com a cooperação da comunidade impossibilita a execução das ações de controle (ABRAHAM et al., 2007; BELLOTO et al., 2011).

Pesquisas de prevalência são fundamentais não só para se avaliar o problema das altas taxas de morbidade relacionadas a essas parasitoses, tal como para criar dados para o planejamento de ações governamentais (ANDRADE et al., 2010). O presente estudo teve por objetivo, avaliar a ocorrência de parasitoses intestinais, através do diagnóstico parasitológico, em crianças assistidas pelo Programa de Erradicação do Trabalho Infantil (PETI).

\section{MATERIAL E MÉTODOS}

O estudo foi conduzido em crianças matriculadas no PETI dos distritos de Interlândia e Sousânia na cidade de Anápolis no ano de 2013. Após a aprovação do estudo pelo comitê de ética da Anhanguera Educacional, sob parecer número 540.734 , os pais e responsáveis pelas crianças matriculadas no projeto foram orientados quanto as propostas do estudo. Aqueles que aceitaram participar assinaram o termo de consentimento livre e esclarecido e responderam um questionário para verificação do perfil socioeconômico e de hábitos de higiene. 
A coleta das amostras foi realizada pelos pais ou responsáveis, utilizando-se frascos coletores universais previamente disponibilizados. O material foi entregue na unidade do PETI e recolhido diariamente de acordo com a demanda. Um total de 48 crianças atenderam todos os requisitos propostos e entregaram as três amostras colhidas em dias alternados. As amostras de fezes foram analisadas no Laboratório Escola de Análises Clínicas da Faculdade Anhanguera de Anápolis-GO. Foi utilizado o método Hoffman de sedimentação espontânea para a busca de enteroparasitas (LOPES et al., 2012). Para as amostras positivas, os pais ou responsáveis foram notificados e orientados a comparecerem em uma unidade de saúde para confirmação do diagnóstico e tratamento.

\section{RESULTADOS E DISCUSSÃO}

A positividade para os enteroparasitos na população estudada foi de $37,5 \%$, o que corresponde a 18 crianças infectadas. Os valores encontrados apresentaram-se acima dos encontrados por BUSNELLO \& TEIXEIRA (2009), que analisaram 44 amostras de crianças de sete a nove anos e encontraram uma positividade de $20 \%$ e por BELO et al. (2012) que realizaram um estudo com 335 alunos de escolas de ensino fundamental no interior do estado de Minas Gerais em que a positividade obtida foi de $29 \%$.

No PETI de Interlândia foram estudadas 29 crianças, destas $21 \%$ obtiveram resultados positivos, em Sousânia foram 19 crianças e $63 \%$ obtiveram resultados positivos (Figura 1).

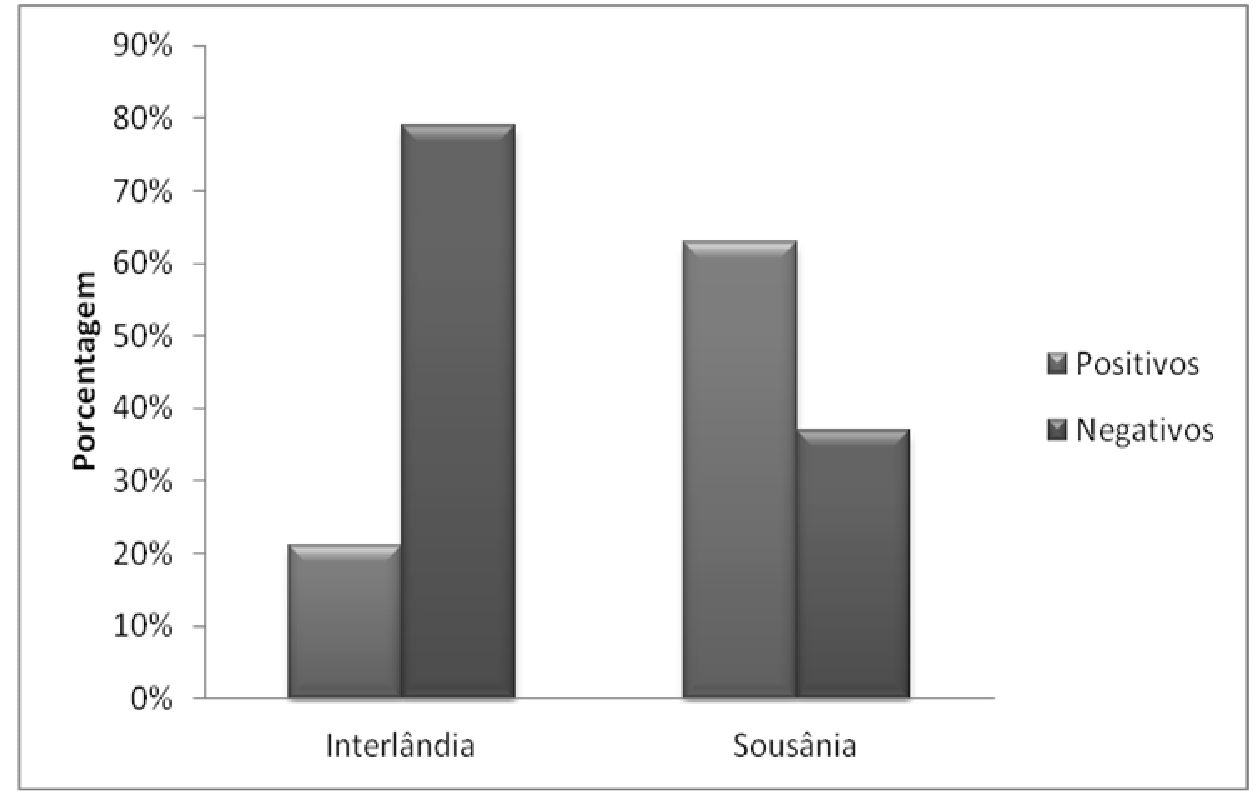

FIGURA 1. Positividade para enteroparasitas nos PETI's de Interlândia e Sousânia no ano de 2013.

Quando avaliados segundo a faixa etária, $62,5 \%$ das amostras positivas para enteroparasitas pertenciam a crianças de cinco a 10 anos e $33,5 \%$ pertenciam a faixa etária de 10 a 14 anos. Resultados semelhantes foram encontrados por SILVA et al. (2010), que estabeleceram a prevalência de enteroparasitoses entre crianças de cinco a 12 anos em Alvorada do Sul-MS e obtiveram 27 positivos na faixa etária de cinco a 10 anos de um número amostral total de 33. 
Quanto a distinção por gênero, das 18 amostras positivas para exames parasitológicos de fezes, notou-se infecção parasitaria em $39 \%$ dos indivíduos do gênero masculino e $61 \%$ para o feminino. De acordo com SANTOS \& MERLINI (2010), independente desta distinção, as afecções parasitárias conseguem alcançar várias populações, com maior ênfase para as crianças menores e que comparecem a escola (período escolar e pré-escolar).

Essa faixa etária é mais vulnerável a infecção parasitária, pois, a prática de lazer de crianças até nove anos são, na maioria das vezes, em recintos externos, à medida que os recintos fechados são mais visitados por crianças maiores. Crianças entre seis a 10 anos têm o hábito de se divertir em jardins ou praças coletivas em convívio com areia. Além disso, crianças apresentam inconscientemente hábitos precários de higiene, como levar as mãos sujas à boca (MATOS \& CRUZ, 2012).

Dentre as 18 amostras positivas nenhuma correspondeu a helmintos, sendo encontrados os protozoários, Entamoeba coli, Endolimax nana, Giardia lamblia e Entamoeba histolytica (Figura 2). Somente 5,5\% apresentavam poliparasitismo, sendo encontrada a associação entre Entamoeba coli e Giardia lamblia. CANTUÁRIA et al. (2011) encontraram resultados similares, neste estudo foram avaliadas 53 amostras e das 21 amostras positivas havia somente a presença de protozoários. A baixa positividade para helmintos pode ser explicada por uma baixa carga parasitaria, baixa contaminação ou uso de antiparasitários empiricamente.

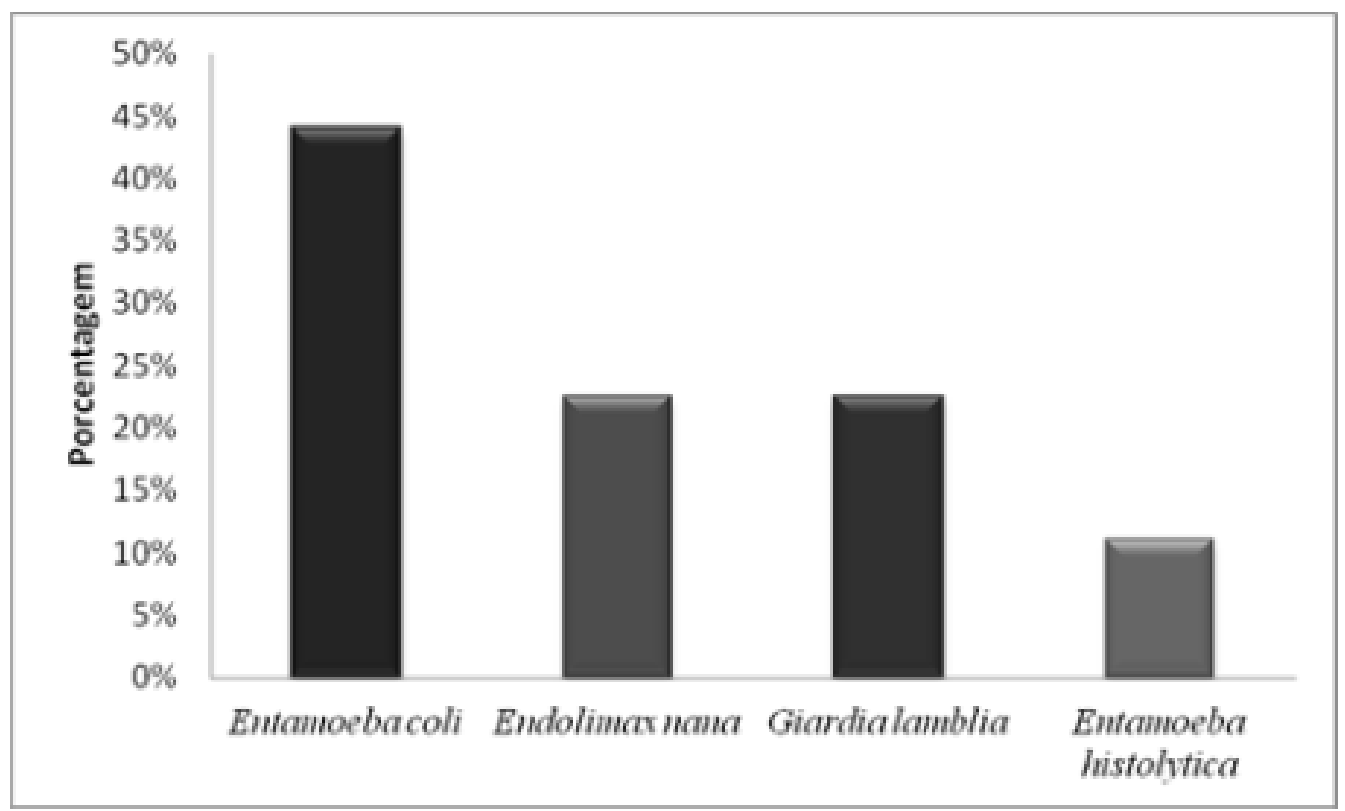

FIGURA 2. Ocorrência de protozoários identificados nas amostras positivas nos PETI's de Interlândia e Sousânia no ano de 2013.

Das famílias estudadas, $29 \%$ relataram que sobrevivem com apenas um salário mínimo, $69 \%$ de um a três salários e $2 \%$ com mais de três salários. As baixas condições financeiras implicam em moradias precárias e hábitos alimentares igualmente precários, o que favorece o aparecimento das enteroparasitoses (SILVA et al., 2010). Em relação às condições higiênico-sanitárias avaliadas em questionário, observou-se que $42 \%$ das residências possuíam água tratada e $56 \%$ 
afirmaram que utilizam água de cisterna. Durante o tratamento da água diversas substâncias químicas são utilizadas para matar os cistos e os ovos dos diversos enteroparasitas. A ausência de água tratada eleva a possibilidade de transmissão dos parasitas intestinais. A água das cisternas pode ser contaminada por material proveniente das fossas, desde que não obedeça a distância mínima estabelecida (ROCHA et al., 2012).

Nos domicílios pesquisados $100 \%$ utilizam fossa, resultado igualmente encontrado por ORO et al. (2010), em que 100\% dos domicílios pesquisados em Descanso Santa Catarina, utilizavam fossa como escoamento dos dejetos. Outro ponto intimamente ligado com predomínio de enteroparasitoses é a forma de expulsão das fezes. A existência de fossa, ou a inexistência de esgoto para o escoamento de fezes, foi preponderante no conjunto de crianças contaminadas com protozoários (VASCONCELOS et al., 2011). A pessoa parasitada contamina o seu próprio ambiente com ovos, larvas e cistos dos enteroparasitos quando não há destino e processamento adequado para os dejetos, visto que, infectará o solo, a água e, portanto os alimentos (NEVES \& MASSARA, 2009).

Apenas $19 \%$ afirmaram ter algum preparo especial para a lavagem das verduras e $64,5 \%$ afirmaram que consomem verduras cruas. A bibliografia nacional tem indicado que a aquisição de mantimentos crus como verduras e frutas com resquícios fecais humanos colaboram para o contágio de várias enteroparasitoses. $\mathrm{A}$ conduta alimentar de adquirir verduras in natura permite a exposição de uma enorme parte dos habitantes às formas de contágio de parasitos (BELLOTO et al., 2011; SILVA et al., 2014).

O consumo de alimentos infectados por parasitos é um dos principais meios de contágio das enteroparasitoses, dessa forma é relevante à precaução em relação aos alimentos lavando-os corretamente, antes de consumi-los, e conservando-os fechados, irrestrito da ação de poeira, mosquitos e quaisquer insetos que sejam capazes de transportar alguma espécie de parasita (MAMUS et al., 2008). Embora $100 \%$ das crianças ingerirem verduras e frutas lavadas, a procedência da água utilizada não assegura que esses alimentos estejam livres de contaminação. O nível de contato com os alimentos já equivale a um fator de risco, além disso manipuladores de alimentos conseguem representar uma possível ameaça de propagação de enteroparasitos caso não apresentem atenção básica (SILVA et al., 2011).

O fato de Sousânia ter apresentado maior índice de positividade em relação à Interlândia pode ser explicado quando avaliam-se os dados encontrados no questionário em relação aos dados obtidos no parasitológico. Apenas $37 \%$ das crianças de Sousânia têm o hábito de lavar as mãos após ir ao banheiro, 32\% costumam lavar as mãos antes das refeições e 74\% consumem verduras cruas.

Já em Interlândia, 48\% tem o hábito de lavar as mãos após ir ao banheiro, $45 \%$ lavam as mãos antes das refeições e $59 \%$ consomem verduras cruas. Grande parte das afecções parasitárias são contraídas por meio da contaminação fecal-oral (LODO et al., 2010; FERNANDES et al., 2014). Algumas medidas devem ser tomadas, tais como a lavagem das mãos após o uso do sanitário, higienização de alimentos, cujo consumo pode ser na forma in natura (verduras, hortaliças) e adotar ações profiláticas para que o número de indivíduos contaminados reduza com o decorrer do tempo (TEIXEIRA et al., 2006).

Os PETI's estudados não oferecem nenhum programa de incentivo para medidas profiláticas, como higienização das mãos antes das refeições e após 
utilização do banheiro. É fundamental proceder com ações que incentivem a modificação em relação ao conhecimento, profilaxia e prevenção destas parasitoses, procurando obter e transmitir informação. Nesse contexto, a educação ambiental no domínio das enteroparasitoses tem se apresentado um método competente, com baixo valor e possível de atingir efeitos relevantes e prolongados e a escola como um centro de educação e aprendizagem deve notar os aspectos epidemiológicos das populações em volta, e mapear os elementos de perigo (GOMES et al., 2012; BARBOSA et al., 2013).

\section{CONCLUSÃO}

Os índices de enteroparasitoses obtidos é resposta da fragilidade das circunstâncias de saneamento básico, assim como as situações de moradia e costumes de asseio das crianças estudadas nos distritos de Intêrlandia e Sousânia.

\section{REFERÊNCIAS}

ABRAHAM, R.S.; TASHIMA,N.T.; SILVA,M.A. Prevalência de enteroparasitoses em reeducando da Penitenciária "Maurício Henrique Guimarães Pereira" de Presidente Venceslau-SP. Revista Brasileira de Análises Clínicas., v.39, n.1, p. 39-42, 2007.

ANDRADE, E.C.; LEITE, I. C. G.; RODRIGUES, O. V.; CESCA, M. G. Parasitoses intestinais uma revisão sobre seus aspectos sociais, epidemiológicos, clínicos e terapêuticos. Revista APS., v.13, n.2, p. 231-240, 2010.

ANTUNES, R. M.; ANTUNES, J. V. M.; OLIVEIRA, L. G. A.; BELINELO, V. J.; VIEIRA FILHO, A. S. Prevalência de enteroparasitoses em crianças de um centro escolar de ambiente rural de São Mateus, ES, Brasil. Enciclopédia Biosfera, Centro Cientifico Conhecer-Goiânia, v.7, n.12, p.1-8, 2011.

BARBOSA, R.N.; MOTA; B. A. E.; CAVALCANTI, M. D. B.; BOTÊLHO, M. C. N.; SANTOS, E. M. Enteroparasitas e profilaxia em alunos da zona rural de Serra Talhada- Pernambuco. PERSPECTIVAS online: biológicas \& saúde, v.9, n.3, p.3745, 2013.

BARRETO, T.C.; BARRETO, T. C.; RIBEIRO, K. S.; MARQUES, A. T. Levantamento das principais parasitoses intestinais que acometem crianças da comunidade de Tamarindo em Campos dos Goytacazes-RJ. PERSPECTIVAS online: biológicas \& saúde, v.7 p.53-61, 2012.

BELLIN, M.; GRAZZIOTIN, N. A. Prevalência de Parasitos Intestinais no Município de Sananduva/RS. News Lab., v.104, p. 116-122, 2011.

BELLOTO, M. V. T.; JUNIOR, J. E. S.; MACEDO, E. A.; PONCE, A.; GALISTEU, K. J.; CASTRO, E.; TAUYR, L. V.; ROSSIT, A. R. B.; MACHADO, R. L. Enteroparasitoses numa população de escolares da rede pública de ensino do Município de Mirassol, São Paulo, Brasil. Revista Pan-Amazônica de Saúde, v. 2, n. 1, p. 37-44, 2011. Disponível em: < http://dx.doi.org/10.5123/S217662232011000100004>. doi: 10.5123/S2176-62232011000100004 
BELO, V. S.; OlIVEIRA, R. B.; FERNANDES, P. C.; NASCIMENTOS, B. W. L.; FERNANDES, F. V.; CASTRO, C. L. F.; SANTOS; W. B.; SILVA, E. S. Fatores associados à ocorrência de parasitoses intestinais em uma população de crianças e adolescentes. Revista Paulista de Pediatria., v.30, n.2, p. 195-201, 2012. Disponível em: < http://dx.doi.org/10.1590/S0103-05822012000200007>. doi: 10.1590/S0103-05822012000200007

BUSNELO, M. I.; TEIXEIRA, I. M. Prevalência de enteroparasitas em estudantes de duas escolas de ensino fundamental. Rev. Fac Farm., v.51, n.2, p. 33-35, 2009.

CANTUÁRIA, F. D.; COCCO, J.; BENTO, R. R. L.; RIBRIRO, F. Avaliação de parasitoses intestinais em escolares do ensino fundamental no município de Coração de Jesus em Minas Gerais Brasil. RBAC., v.43, n. 4, p. 277-83, 2011.

FERNANDES, N. S.; GUIMARÃES, H. R.; AMORIM, A. C. S.; BRITO, V. M.; BORGES, E. P.; REIS, M. B.; TRINDADE, R. A.; MELO, A. C. F. L. Ocorrência de enteroparasitoses em manipuladores de alimentos de restaurantes em Parnaíba, Piauí-Brasil. Rev. patol. trop, v. 43, n. 4, p. 459-469, 2014. Disponível em: < http://dx.doi.org/ 10.5216/rpt.v43i4.33614>. doi: 10.5216/rpt.v43i4.33614.

FREI, F.; JUNCASEM, C.; PAES, J. T. R. Levantamento epidemiológico das parasitoses intestinais: viés analítico decorrente do tratamento profilático. Caderno de saúde.v.24, n.12, p.2919-2925, 2008.

GELATTI, L. C.; PEREIRA; A. S. S.; MENDES, A. P. S.; JASEM, D. F. A.; NASCIMENTO, F. S.; BASTOS, H. L.; SOUZA, M. F.; PAULA, M. B. C.; SILVA, M. V. S.; REIS, N. O. Ocorrência de parasitos e comensais intestinais numa população de escolares do município de Uruaçu, GOIAS. Revista Fasem Ciências. v.3, n.1, p.55$65,2013$.

GOMES, P.G.; SOUZA, J.B.; SOUZA, T.S. Trabalho pedagógico realizado no PETI: Desafios e perspectivas de uma atuação multifacetada na contemporaneidade. Práxis Educacional. Vitória da Conquista, v.8, n. 13 p.129-142, 2012.

GOMES, S. C. S.; REZENDE, L. P.; SANTOS, R. S.; IVES, D. O.; SILVA, E. A. Prevalencia de enteroparasitos em humanos residentes em uma comunidade do municipio de Grajau-MA. Pesquisa em Foco, v.19, n.1, p. 53-62,2011.

LODO, M.; OLIVEIRA, C. G. B.; FONSECA, A. L. A.; CAPUTTO, L. Z.; PACKER, M. L. T.; VALENTI, V. E.; FONSECA, F. L. A. Prevalencia de enteroparasitas em municipio do interior Paulista. Revista Brasileira de Crescimento e Desenvolvimento Humano., v.20, n. 3, p.769-777, 2010.

LOPES, C. R.; SALAMAIA, F. H.; MOLINARI, L. M. Diferentes parasitos intestinais em crianças de um a dez anos atendidas em um laboratório de análises clínicas na cidade de marialva, Paraná, Brasil. Revista Saúde e Pesquisa. v.5, n.2, p.290297,2012 . 
MAMUS, C. N. C.; MOITINHO, A. C. C.; GRUBE, C. C.; MELO, E. M.; WEILER, E. B.; ABREU, C. A.; BELTRÃO, L.; SOARES, P. B.; BELTRAME, S.; RIBEIRO, S.; ALEIXO, D. L. Enteroparasitoses em um centro de educacao infantil do municipio de Iretama/PR. Revista de Saúde e Biologia. v.3, n.2, p.39-44, 2008.

MATOS, M. A.; CRUZ, Z. V. Prevalência das parasitoses intestinais no município de Ibiassucê-Bahia. REMAS., v.5 n. 1, p.64-71, 2012.

MELO, E. M.; FERRAZ, F. N.; ALEIXO, D. L. Importância do estudo da prevalência de parasitos intestinais de crianças em idade escolar. Revista Saúde e Biologia., v.5, n.1, p.43-47, 2010.

MONROE, N. B.; LEITE, P. R. R.; SANTOS, D. N.; SÁ-SILVA, J. R. O tema transversal saúde e o ensino de ciências: representações sociais de professores sobre as parasitoses intestinais. Investigações em Ensino de Ciências, v.18, p.722, 2013.

MORAIS NETO, A. H. A. ; SANTOS, C.P.; ALMEIDA, J.C.A. Uma reflexão sobre as parasitoses intestinais em comunidades de baixa renda do norte do Estado do Rio de Janeiro, Brasil. Revista Práxis. v.1 n.2, p.71-74, 2009.

NEVES, R. L. S.; MASSARA, C. L. Contaminação do solo de áreas contaminadas do município de Caratinga, MG, por ovos de Toxocara sp. e cistos de Entamoeba sp. Revista de Patologia Tropical, v.38, n.2, p. 126-130, 2009.

ORO, D.; KOPOROSKI, G. K.; ORO, N. A.; SBARDELOTTO, C.; SEGER, J. Prevalencia de parasitas intestinais em criancas de Descanso-Santa Catarina-Brasil. Unoesc \& Ciência- ACBS, v.1, n.2, p.151-156, 2010.

ROCHA, R. A. P.; PÁGIO, R. B.; MIRANDA, A. E. B.; PEREIRA, F. E. L.; MACIEL, E. L. N. Determinantes das parasitoses intestinais em população infantil de assentamentos rurais do município de Alegre, ES. Revista Brasileira de Pesquisa em Saúde. v.14 n.1 p.26-35, 2012.

SANTOS, A. S.; MERLINI, L. S. Prevalência de enteroparasitose na população do município de Maria Helena, Paraná. Ciência e Saúde Coletiva., v.15, n.3, p.899905, 2010.

SILVA, A. M. B.; BOUTH, R. C.; COSTA, K. S.; CARVALHO, D. C.; HIRAI, K. E.; PRADO, R. R.; ARAÚJO, S. G.; PEREIRA, A. C. L.; RIBEIRO, K. T. S. Ocorrência de enteroparasitoses em comunidades ribeirinhas do Município de Igarapé Miri, Estado do Pará, Brasil. Revista Pan-Amazônica de Saúde, v. 5, n. 4, p. 45-51, 2014.

SILVA, F. M. A.; LIMA, M. I. S.; SOUZA, T. A.; ALVES, E. V. C.; FIALHO, E. M. S.; ALMEIDA, B.; DINIZ, J. P.; BORGES, R. R.; MUNIZ, D. B.; BASTOS, A. C. S. C.; FIGUEIRO, T. A. P.; MELO, O. T.; NASCIMENTO, A. R.; AZEVEDO, G. G. Aspectos epidemiológicos e prevalência de enteroparasitoses em crianças do bairro Jambeiro, SÃO LUÍS, MA. Rev. Ciênc. Saúde., São Luís, v.13, n.2, p. 123-130, 2011 a. 
SILVA, J. C.; FURTADO, L. F. V.; FERRO, T. C.; BEZERRA, K. C.; BORGES, E. P.; MELO, A. C. F. L. Parasitismo por Ascaris lumbricoides e seus aspectos epidemiológicos em crianças do Estado do Maranhão. Revista da Sociedade Brasileira de Medicina Tropical., v.44 n.1 p.100-102, 2011b. Disponível em: < http://dx.doi.org/10.1590/S0037-86822011000100022>. doi: 10.1590/S003786822011000100022

SILVA, J.E.C.; PARENTE, B.; BURGOS, V.O. Prevalência de parasitas intestinais em crianças de 05 a 12 anos em nova Alvorada do Sul-MS. Interbio., v.4, n.1, p. 514, 2010.

TEIXEIRA, M.E.; FLORES, R.E., FUENTEFRIA A.M. Prevalência de enteroparasitas em crianças de uma creche na cidade de Concórdia, Santa Catarina, Brasil. Newslab., v.78, 2006.

VASCONCELOS, I.A.B.; OLIVEIRA, J. W.; CABRAL, F. R. F.; COUTINHO, H. D. M.; MENEZES, I. R. A. Prevalência de parasitoses intestinais entre crianças de 4-12 anos no Crato, Estado do Ceará: um problema recorrente de saúde pública. Acta Scientiarum. Health Sciences, v.3, n.1, p.35-41, 2011. Disponível em: <http://dx.doi.org/10.4025/actascihealthsci.v33i1.8539>. doi: 10.4025/actascihealthsci.v33i1.8539 